\title{
Patterns of exposure to SARS-CoV-2 carriers manifest multiscale association between urban landscape morphology and human activity
}

\section{Gabriel I. Cotlier}

University of Haifa

Yoav Lehahn ( $\nabla$ ylehahn@univ.haifa.ac.il)

University of Haifa

Doron Chelouche

University of Haifa

\section{Research Article}

Keywords: urban remote sensing, urban landscape, landscape patchiness, human activity, satellite nighttime lights, COVID-19, SARS-CoV-2 carriers.

Posted Date: September 16th, 2020

DOI: https://doi.org/10.21203/rs.3.rs-77773/v1

License: (a) (1) This work is licensed under a Creative Commons Attribution 4.0 International License.

Read Full License

Version of Record: A version of this preprint was published at Scientific Reports on November 11th, 2021.

See the published version at https://doi.org/10.1038/s41598-021-01257-8. 


\title{
Patterns of exposure to SARS-CoV-2 carriers manifest multiscale association between urban landscape morphology and human activity
}

\author{
Gabriel I. Cotlier ${ }^{1}$, Yoav Lehahn ${ }^{2 *}$, Doron Chelouche ${ }^{1}$ \\ ${ }^{1}$ Haifa Center for Theoretical Physics and Astrophysics (HCTPA), and The Data Science \\ Research Center (DSRC), University of Haifa, Haifa 3498838, Israel. \\ ${ }^{2}$ Department of Marine Geosciences, Charney School of Marine Sciences, University of Haifa, \\ Haifa 3498838, Israel. ${ }^{*}$ Corresponding author's email: ylehahn@univ.haifa.ac.il
}

\begin{abstract}
The outbreak of the Coronavirus disease 2019 (COVID-19), and the drastic measures taken to mitigate its spread through imposed social distancing, have brought forward the need to better understand the underlying factors controlling spatial distribution of human activities promoting disease transmission. Focusing on results from 17,250 epidemiological investigations performed during early stages of the pandemic outbreak in Israel, we show that the distribution of carriers of the respiratory severe acute syndrome coronavirus-2 (SARS-CoV-2), which causes COVID-19, is spatially correlated with two satellite-derived surface metrics: night light intensity and landscape patchiness, the latter being a measure to the urban landscape's scale-dependent spatial heterogeneity. We find that exposure to SARS-CoV-2 carriers was significantly more likely to occur in "patchy" parts of the city, where the urban landscape is characterized by high levels of spatial heterogeneity at relatively small scales $(\sim 10-100 \mathrm{~m})$. We suggest that this spatial association reflects a scale-dependent constraint imposed by the city's morphology on the cumulative behavior of the people inhabiting it. The presented results shed light on the complex interrelationships between humans and the urban landscape in which they live and interact, and open new avenues for implementation of multi-satellite data in large scale modeling of phenomena centered in urban environments.
\end{abstract}

Key Words: urban remote sensing; urban landscape; landscape patchiness; human activity; satellite nighttime lights; COVID-19; SARS-CoV-2 carriers. 


\section{Introduction}

More than $50 \%$ of the world's population presently lives in cities, with an estimate forecast of the urban population in 2050 being around 10 billion $^{1}$, making human health and well-being increasingly dependent on the dynamics of the urban environment. Current demographic trends, driven by rapid urbanization, migration flows, deforestation, climate change, inequality and political instability have affected the dynamics of infectious diseases and catalyzed the transmission of epidemic outcracks at global scale ${ }^{2}$. The increase in urbanization can function as an incubator for the spread of emerging zoonotic disease and epidemics ${ }^{3}$, and intra-urban crowding tends to promote the spread of infectious diseases ${ }^{4}$. The increase of urban agglomeration ${ }^{5}$, leading to a globally connected high population density, reinforces the need to better understand the possible impact of spatial changes in urban landscape on public health and in particular on the spread of diseases ${ }^{6}$.

Epidemiology and its association with spatial patterns of landscape and human activity have received much attention with the recent outbreak of the severe acute respiratory syndrome coronavirus 2 (SARS-CoV-2) pandemic, which causes the corona virus disease (COVID-19). Spatial data of COVID-19 incidence rates at county-level across the US, reveal a strong positive relationship with income inequality ${ }^{7}$. Network connectivity approach was applied to VIIRS and census data to quantify spatiotemporal evolution of SARS-CoV-2 epidemic propagation in the US; the findings indicate that spatiotemporal evolution of the epidemic may be quantified by analyzing transportation networks of different connectivity levels ${ }^{8}$. Satellite nighttime light (NTL) radiance and Air Quality Index data have been used to characterize spatiotemporal changes of human activity before and during the pandemic in the US, showing that NTL radiance increased in residential areas and decreased in commercial centers after government lockdown was imposed, while transportation and public facilities seem to remain unaltered ${ }^{9}$. Employing mobile data tracking, a large increase in the daily usage of urban green areas by pedestrians and cyclists, who aim to preserve social distancing, was found in Oslo, Norway ${ }^{10}$. A body of research employing satellite observations has focused on analyzing the impact of SARS-CoV-2 epidemic restrictions on mobility and industry often showing reduction in anthropogenic emissions and improvement in air quality ${ }^{11,12}$.

Following the large body of work on the spatial aspects of disease spread in general, and of COVID-19 in particular, here we address the important, yet poorly investigated question of whether predictable relationships can be found between urban landscape, human activity, and patterns of exposure to SARS-CoV-2. Specifically, we hypothesize that patterns of heterogeneity in the urban landscape impose spatially varying constraints on interpersonal proximity and crowding conditions, thus affecting the potential for the spread of COVID-19. To test this hypothesis, we investigate the spatial association between patterns of exposure to SARS-CoV-2 carriers, with satellite-based landscape-heterogeneity measure, and nightlight intensity data. As a case study we focus on Israel, however we gave attention to Gush Dan District as well as consider a selected sample of 30 cities, during a one-month period of intense COVID-19 outbreak, between March and April 2020. We further explore in some detail the largest conurbation in Israel, named Gush Dan, which includes the metropolitan area of Tel-Aviv and is home to about 50\% of Israel's population. 


\section{Results}

Characterizing the spatial association between patterns of exposure to SARS-CoV-2 and urban landscape heterogeneity, requires an effective representation of the two components. According to the "patch-mosaic model", landscape is composed of a structure of discrete units (or patches) assumed to have a relative inner uniformity and sharply defined boundaries which conform mosaics (or aggregation patches) ${ }^{13,14}$. Patchiness measures have long been used to characterizing natural landscapes ${ }^{15,16,17}$, as well as for studying urban environments ${ }^{18,19,20}$. In order to accurately capture landscape heterogeneity across multiple scales, the definition of measures based on a continuous signal enables to capture the potential loss of information resulting from dividing the landscape into separate categorical units ${ }^{21,22,23}$. Several continuous spatial indices ${ }^{24}$ were developed to analyze urban landscape heterogeneity, including approaches such as spatial autocorrelation $^{25}$, Fourier analysis ${ }^{26}$, and wavelet transform ${ }^{27}$ among others. Here, for grasping landscape heterogeneity across scales reducing potential loss of information ${ }^{28}$ we apply a surfacebased metric — the landscape patchiness index (LPI) — based upon scale variance model. The LPI depicts the distribution of the power spectrum through pixels in a continuous scale, with high LPI values representing more "patchy" landscapes, with substantial variance at small spatial scales (see details in the Methods section).

The ability of the LPI to characterize multi-scale spatial patterns in the urban landscape, is exemplified by comparing randomly selected $480 \mathrm{~m}$ by $480 \mathrm{~m}$ Landsat images of urban areas characterized by different LPI levels (Fig. 1). Higher values of LPI, associated with high variance on small scales are often characteristic of highly developed urban regions, with relatively narrow streets in between the buildings, whereas lower values of LPI imply smoother landscapes, with less variance on small scales (compare lower and upper panels in Fig. 1, respectively). Note that the observed patchiness may also depend on seasonal effects (e.g., the presence of vegetation or lack thereof), as well as on the illumination angle of a non-smooth terrain (e.g., the casting of shadows by buildings, hills, and creeks). We find that although patchiness values are wavelengthdependent, the trends obtained here are robust. 


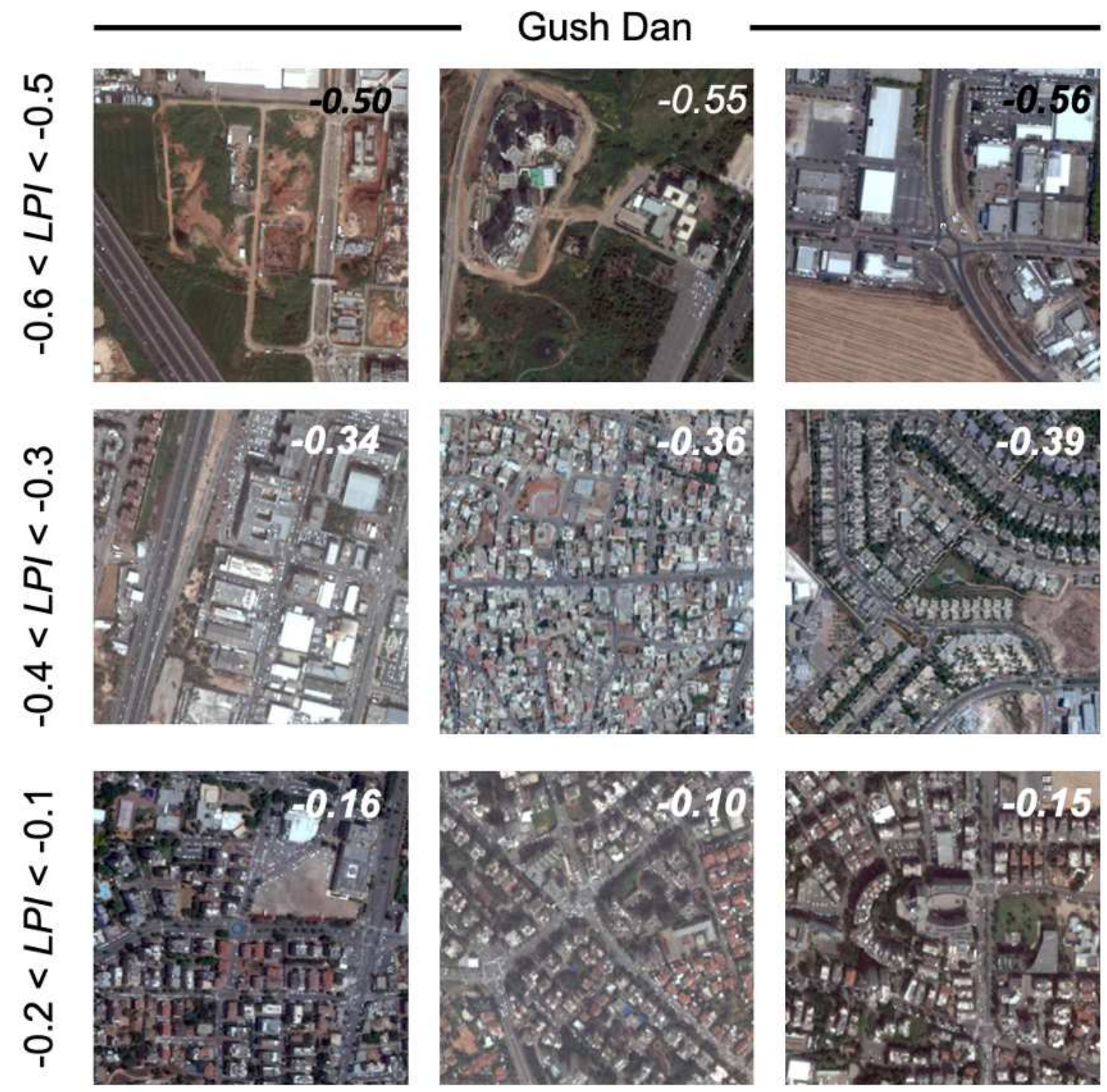

Figure 1. Examples of the variability of landscape types across different ranges captured by LPI for the area of Gush Dan District. It is shown a true color image of LPI ranges within the interval $-0.1<$ LPI $<$ 0.6 which demonstrates the captured differences in urban landscape heterogeneity.

Complementary information on the spatial characteristic of the urban landscape and its expression in patterns of human activity is obtained by the nighttime light signal (NTL). The NTL is commonly used in urban studies, and has been associated with socioeconomic dynamics ${ }^{29}$, population density ${ }^{30}$, urbanization $^{31}$, crime analysis $^{32}$, migrations ${ }^{33}$, military conflicts ${ }^{34}$, spread of epidemics ${ }^{8}$ identification of commercial areas ${ }^{35}$, tourism ${ }^{36}$ among many others, constituting a good predictor of the magnitude of human activity. 
Spatial characteristics of reported locations of SARS-CoV-2 carriers (RLSC) between 10/03/2020 and 14/04/2020 corresponding to the initial wave of COVID-19 were analyzed from the public spatiotemporal database compiled by the Israeli Ministry of Health ${ }^{37}$. The database consists of the results of epidemiological investigations, showing known locations of SARS-CoV2 carriers up to two weeks prior to their diagnosis (after being diagnosed SARS-CoV-2 carriers have been immediately isolated). For the period covered by this study, the database consists of 17,250 reported RLSCs. To characterize the RLSC locations, we classify them into 7 categories of human activity according to the information provided in the epidemiological investigation, based on a randomly selected sub-sample of $10 \%$ of the total RLSC data set (Fig. 2). The largest amount (39\%) of RLSCs were categorized as religion-related (including synagogues, Jewish religious studding centers, traditional baths, etc.); followed by recreational venues $(17 \%$, including culture establishments, restaurants, bars, coffee shops, sports centers, youth clubs, playgrounds, etc.); commercial grounds (13\%, including different kinds of shops such as cloth; food; computers; cellphones; etc., as well as commercial services); supermarkets and grocery stores (11\%, including small local to large chains); health facilities, $(10 \%$ including clinics, hospitals, pharmacies, etc.); and education establishments (6\%, including from kindergartens to universities, language school, research centers, etc.).

\section{SARS-CoV-2 Exposures based on Human Activity type}

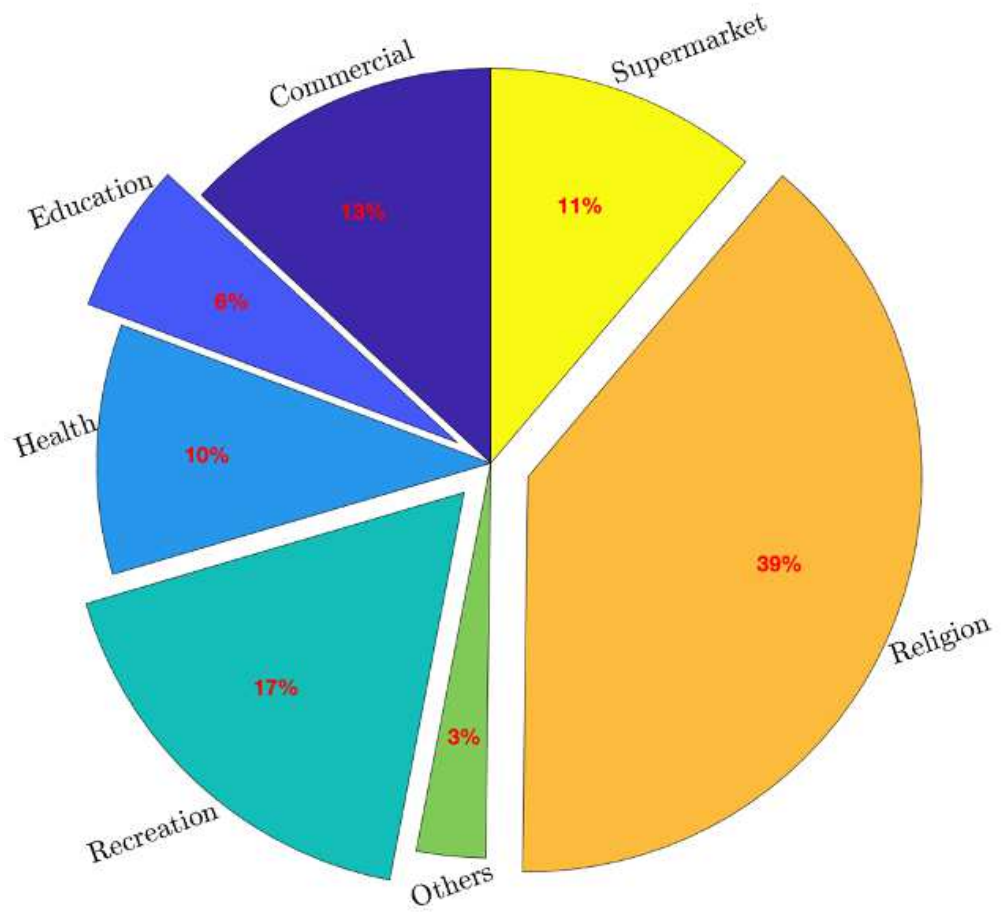

Figure 2. Pie chart describing the classification of RLSCs into human activity types.

To further investigate the factors underlying spatial distribution of RLSC we compare it with the spatial characteristics of surface measures extracted from satellite data. We first focus our 
analysis on the Gush Dan District, which is a densely populated area including Tel Aviv city and its metropolitan area. It has total population of approximately about 4 million residents, comprising about $44 \%$ of the population of Israel ${ }^{38}$ (Fig. 3). The dependence of RLSC distribution on landscape morphology can be qualitatively observed in a true colors satellite image, which emphasizes the fact that RLSC data points are mostly found in highly developed urban areas (Fig. 3a). In agreement with that, RLSCs are mostly found in areas of high NTL levels, with mean values of $72.166 \pm 0.574\left[\mathrm{nWcm}^{-2}\right]$. For comparison, the average NTL level in regions with no reported RLSCs is $19.78 \pm 0.273$ [nWcm ${ }^{-2}$ ] (Fig. 3b). Interestingly, a tracer to RLSC distribution in space is also provided by the LPI, which is characterized by average values of $-0.343 \pm 0.007$ and -0.522 \pm 0.002 , for grid cells containing and not containing RLSC data points, respectively (Fig. 3c).
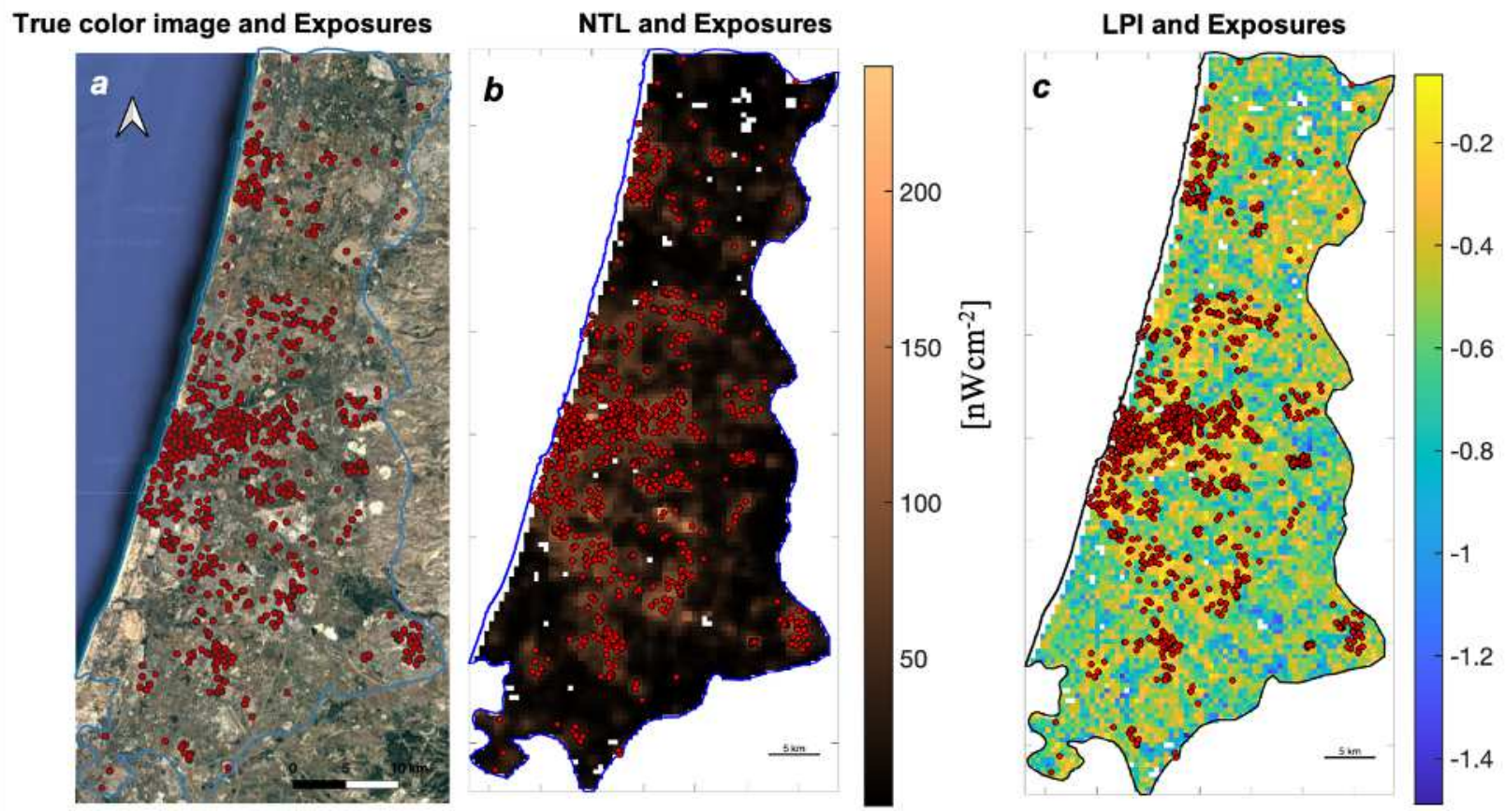

Figure 3. Spatial distribution of RLSC in Gush Dan District. RLSC in Gush Dan (red points), overlaid on (a) a true-color satellite image (Gush Dan borders in blue); (b) NTL map; (c) LPI map. Note that RLSCs preferentially occur in regions with high LPI values, which correspond to regions with high NTL values.

Following this relatively coarse analysis, which largely reflects distinction between urban and non-urban landscapes, we next probe the landscape patterns underlying RLSC spatial distribution at the city scale. To this end, we first compare average NTL and LPI values at locations with RLSCs to those values at locations without RLSC. We do so for the 30 cities reported by the Israeli Ministry of Health to have more than 100 RLSCs (Fig. 4). We find that average LPI and NTL values are significantly higher at locations with reported RLSCs in $77 \%$ and $90 \%$ of the cities, respectively. This demonstrates that LPI, like NTL, can be used as a tracer to intense human activity within the urban landscape. 


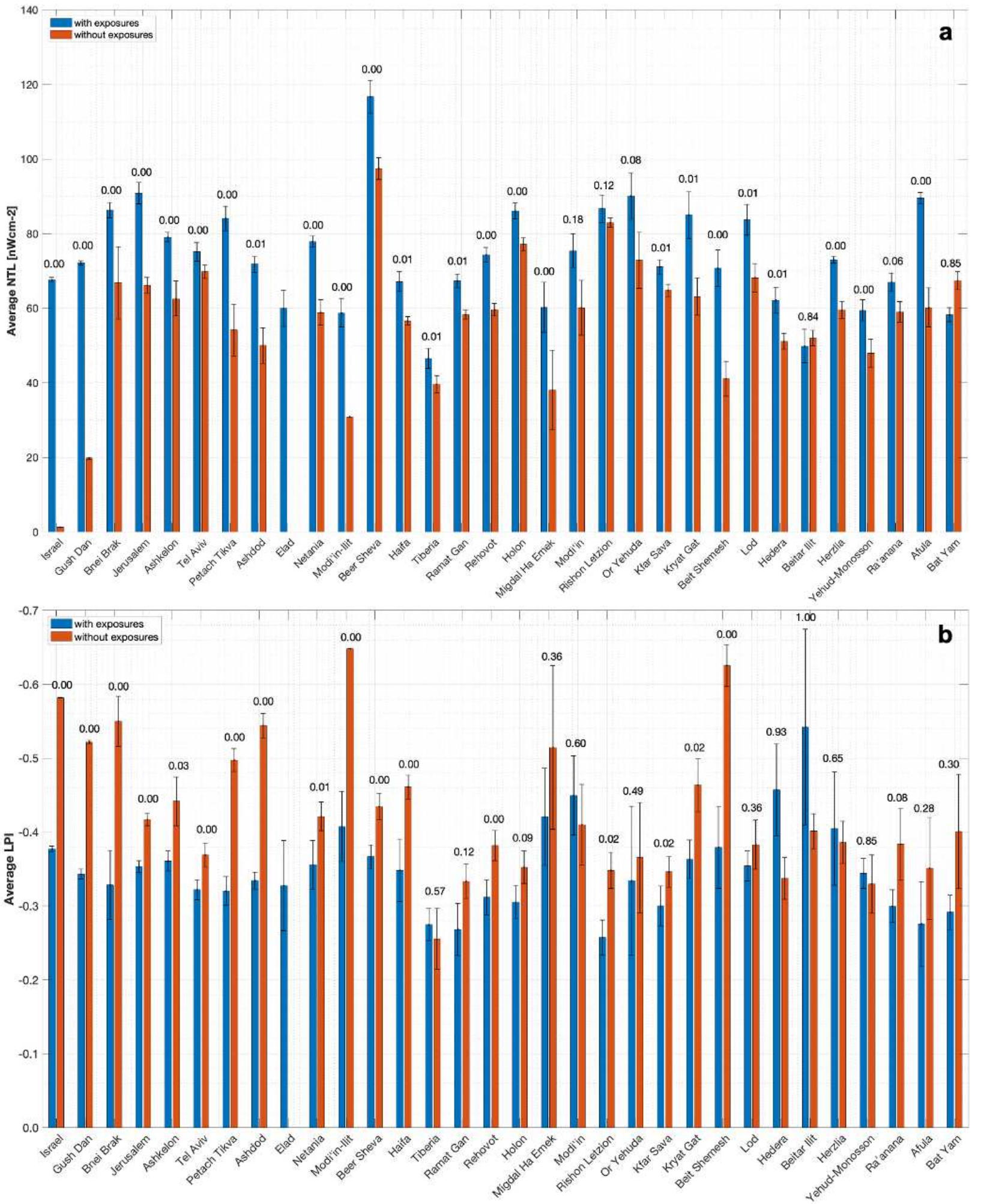

Figure 4. NTL and LPI statistics for our sample. Bar plot showing the mean of (a) NTL and (b) LPI in grid cells with exposures (blue bars) and without exposures (red bars) for the 30 cities with more than 100 reported RLSC cases. For comparison we also present the statistics for the full sample of cities, as well as that for the Gush Dan conurbation. Note that in almost all of the cities both NTL and LPI are significantly higher at locations with finite RLSC values. 
The association between the RLSC and the spatial characteristics of the urban landscape is emphasized when considering the median RLSC values for locations as a function of LPI/NTL (the LPI/NTL range is divided into bins, and the median RLSC is calculated for all locations corresponding to those bins). Results for the entire area covered by the Ministry of Health reports are shown in Fig. 5. Evidently, similar behavior is found for the NTL and for the LPI, whereby higher values, which correspond to more developed areas are associated with a higher (median) RLSC values. Interestingly, there is little dependence of the median RLSC on the LPI or the NTL for low values in those properties. In contrast, a much steeper relation is observed for higher values of NTL, LPI, and RLSCs. The similar behavior of NTL and LPI statistics further demonstrates that LPI, like NTL, is a tracer of human activity and development.

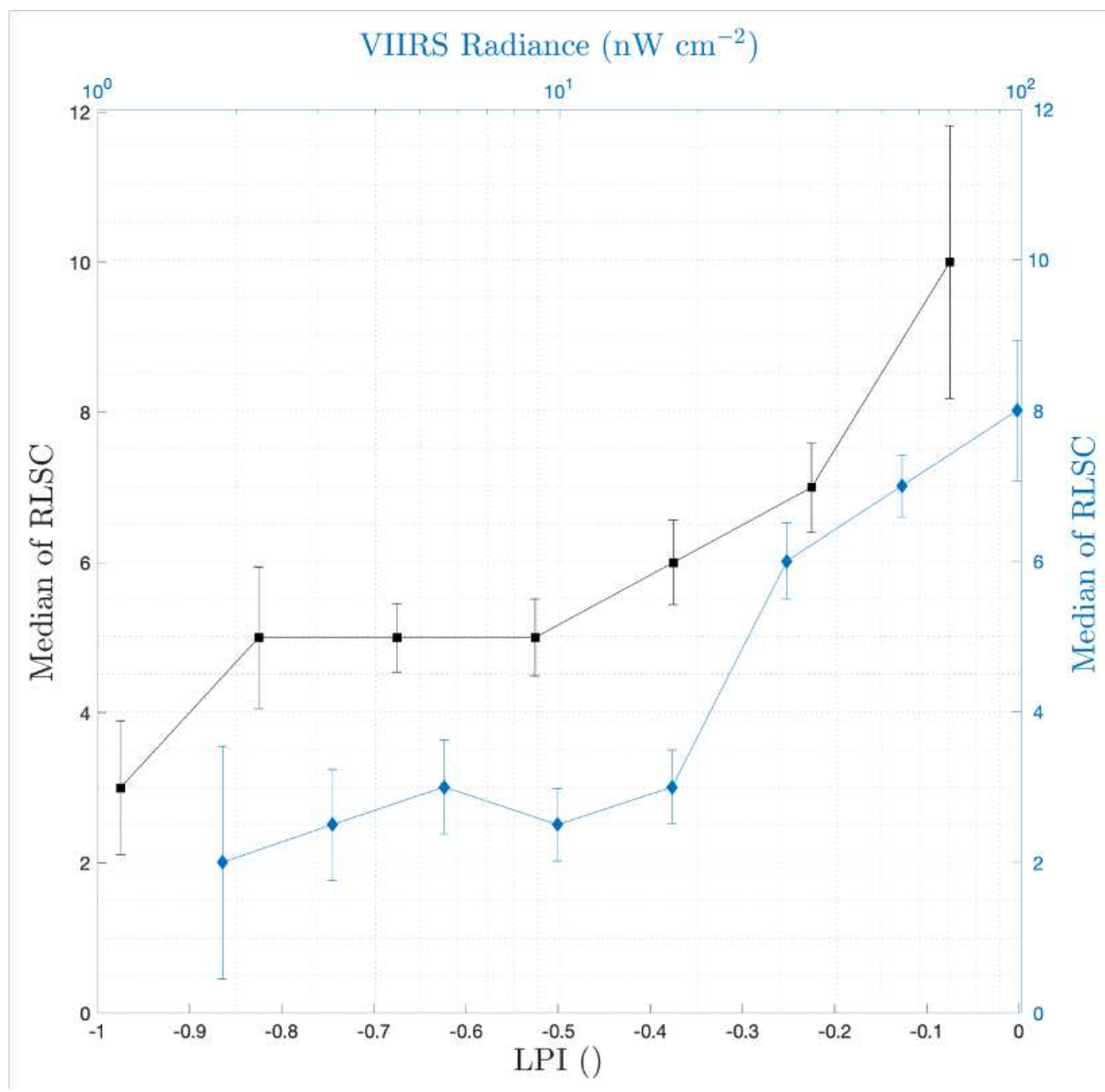

Figure 5. Correlation between RLSC and NTL (blue curve) and LPI (line curve). Dots and error-bars denote the median and standard deviation in each bin. Note the qualitatively similar behavior for the NTL and LPI statistics demonstrating the commonalities between LPI and NTL as tracers of human activity and landscape development. 


\section{Conclusions}

Using an integrated dataset comprised of satellite observations and results from epidemiological investigations, we explore the interrelationships between spatial characteristics of urban landscape, human activity, and distribution patterns of reported locations of SARS-CoV-2 carriers, or RLSC, in a highly populated region in the state of Israel. Our results show that at least for the time and location covered in this study, satellite-based levels of NTL and LPI are statistically significant measures to the likelihood of a given cell within the urban area to host a RLSC. While linkages between NTL and different aspects of human activity were previously investigated in numerous studies $29,30,31,32,33,34,35,36$ to the best of our knowledge association between spatial patterns of human activity, and satellite-derived urban landscape heterogeneity, as expressed here by the distribution of RLSC and levels of LPI, respectively, is shown here for the first time.

This research strongly relies on epidemiological investigations that are meant to track trajectories SARS-CoV-2 carriers prior to being diagnosed, in order to identify potential cases of exposure and, ultimately, stop the chain of infection. It is likely to assume that spatial distribution patterns of RLSC emerging from the investigation are strongly linked to patterns of human agglomeration and reflect pathways of disease spread. Accordingly, the spatial association between RLSC and NTL can be explained by the fact that higher values of the latter simply represent areas of intense human activity, where encounters between people are more frequent.

While NTL values are directly linked to the level of human activity, LPI captures the spatial characteristics of the urban landscape in which the human activity takes place. We suggest that the spatial association between LPI and RLSC reflects a fundamental scale-dependent constraint imposed by the morphology of the city's landscape on the cumulative behavior of the people inhabiting it. Accordingly, patchy (i.e. high LPI) sections of the city, in which landscape variance becomes important at small spatial scales (a few to tens of meters) that resonate with typical scales of human interactions, are more prone to human agglomeration and crowding, and thus to decease spread. In contrast, areas of homogeneous landscape, with only little contribution to the overall landscape variance from small scale patterns, encounters between people are, in general, less frequent. Consequently, as observed here, events of exposure to SARS-CoV-2 carriers are significantly more likely to occur in patchy parts of the city.

In addition to improving our understanding on the interrelationship between humans and the urban landscape in which they live and interact, the unique observations presented in this work open new avenues for implementing multi-satellite data, as the ones used here, in large scale modeling of phenomena centered in urban environments. Such implementation is likely to improve human society's ability to predict, and potentially mitigate, the spread of diseases as the COVID19.

\section{Methods}

\section{Epidemiological data}

Spatial characteristics of reported locations of SARS-CoV-2 carriers (RLSC) between $10 / 03 / 2020$ and 14/04/2020 were analyzed from the public spatiotemporal database compiled by the Israeli Ministry of Health ${ }^{37}$. The database consists of the results of epidemiological 
investigations, conducted on positively diagnosed SARS-CoV-2 patients. The database was updated on a daily basis, with results available online for a two weeks period. From this database we used georeferenced information on locations of SARS-CoV-2 carriers to two weeks prior to their diagnosis.

\section{Extraction of the Landscape Patchiness Index (LPI)}

We introduce a modified version of an analytical method based on the so-called "geographic variance" or "scale variance" approach, first developed by ${ }^{39}$ and further applied by ${ }^{40}$,

${ }^{41}$ for describing the formation and spatial dynamics of landscape heterogeneity across scales ${ }^{42}$. Employing a nested hierarchy of levels across multiple scale enables to capture each level's independent contribution to the total system's variance. Different versions of this method haven been applied in different disciplines such as oceanography ${ }^{43}$, and economic geography ${ }^{44}$. Here we employed a mosaic composed of three Landsat 8 Level-2 surface reflectance images downloaded from USGS Earth Explorer ${ }^{45}$, corresponding to the green visible range $(0.525-0.600 \mu \mathrm{m})$ and having a native spatial resolution scale, $l_{0}=30 \mathrm{~m}$. The scale-dependent variance, $\sigma_{n}^{2}$ is calculated within sub-image tiles of size $l_{n}=2^{n} l_{0}$, with $n=1,2,3,4$ so that the maximum tile-size considered is $l_{\max }=480 \mathrm{~m}$, which is comparable to the VIIRS night-light image resolution ${ }^{46}$. The calculation of the LPI proceeds as follows: the scale-dependent mean variance, $\left\langle\sigma_{n}^{2}\right\rangle$ is obtained by averaging over $\sigma_{n}^{2}$-values for all the sub-image tiles of size $l_{n}$ that are within in a given tile of size $l_{\max }$. A powerlaw fit to the scale-dependent variance, $\left\langle\sigma_{n}^{2}\right\rangle \propto n^{-p}$ is then obtained, where $p$ corresponds to the LPI at that location. Higher values of $p$ imply substantial variance on small scales, which often characterizes developed regions, where structures are of order human scales, which are $\ll l_{\max }$ (see examples in Fig.1). Conversely, lower values of $p$ imply smoother landscapes.

\section{Satellite Data}

The LPI is derived from Landsat 8 Level-2 surface reflectance images of the 31 of July 2017 downloaded from USGS Earth Explorer ${ }^{45}$, corresponding to the green visible range (0.525$0.600 \mu \mathrm{m}$ ) with $30 \mathrm{~m}$ of spatial resolution. Nighttime light intensity is derived from monthly average radiance composite data of July 2017 from Suomi National Polar-orbiting Partnership (SNPP) VIIRS Day-Night Band (DBN) downloaded from Google Earth Engine ${ }^{47}$ with an estimated spatial resolution of $15 \times 15$ asec $^{2}$ which roughly corresponds to $460 \times 460 \mathrm{~m}^{2}$ for the latitude of study.

Author contribution G.I.C, Y.L and D.C. conceived and designed the study. G.I.C conducted the data processing and analysis. G.I.C, Y.L and D.C. interpreted the results and wrote the paper.

Competing interests The authors declare no competing interests.

Data Availability Israeli Ministry of Health geodatabase is freely available at https://imoh.maps.arcgis.com/apps/webappviewer/index.html?id=20ded58639ff4d47a2e2e36af464c36e\&locale=he \&/ Landsat 8 data is freely available from USGS Earth Explorer at https://earthexplorer.usgs.gov VIIRS data is freely available at https://developers.google.com/earthengine/datasets/catalog/NOAA_VIIRS_DNB_MONTHLY_V1_VCMCFG 


\section{Reference}

1. Kaneda, T. PRB's 2016 World Population Data Sheet. http://www.prb.org/Publications/Datasheets/2016/2016world-population-data-sheet.aspx (2016).

2. Bedford, J. et al. A new twenty-first century science for effective epidemic response. Nature 575(7781), 130-136 (2019).

3. Neiderud, C.-J. How urbanization affects the epidemiology of emerging infectious diseases. Infection Ecology \& Epidemiology 5(1), 27060 (2015).

4. Dye, C. Health and Urban Living. Science 319(5864), 766-769 (2008).

5. Fang, C. \& Yu, D. Urban agglomeration: An evolving concept of an emerging phenomenon. Landscape and Urban Planning 162, 126-136 (2017).

6. Lee, V.J. et al. Epidemic preparedness in urban settings: new challenges and opportunities. The Lancet Infectious Diseases 20(5), 527-529 (2020).

7. Mollalo, A., Vahedi, B. \& Rivera, K.M. GIS-based spatial modeling of COVID-19 incidence rate in the continental United States. Science of The Total Environment 728, 138884 (2020).

8. Small, C., MacDonald, A. J, \& Sousa, D. Spatial network connectivity of population and development in the USA; Implications for disease transmission. Preprint at https://arxiv.org/abs/2004.14237v1 (2020).

9. Liu, Q. et al. Spatiotemporal Patterns of COVID-19 Impact on Human Activities and Environment in Mainland China Using Nighttime Light and Air Quality Data. Remote Sensing 12(10), 1576 (2020).

10. Venter, Z. S., Barton, D.N., Gundersen, V., Figari, H; Nowell M. Urban nature in a time of crisis: recreational use of green space increases during the COVID-19 outbreak in Oslo, Norway. Preprint at https://doi.org/10.31235/osf.io/kbdum (2020).

11. Chauhan, A. \& Singh, R.P. Decline in PM2.5 concentrations over major cities around the world associated with COVID-19. Environmental Research 187, 109634 (2020).

12. Kasturi, D. K., Kamarul Zaman N. A. F., Kaskaoutis, D. G., Latif, M. T. COVID-19's impact on the atmospheric environment in the Southeast Asia region. Science of the Total Environment 736, 139658 (2020).

13. Forman, R.T.T. Land mosaics: the ecology of landscapes and regions. (Cambridge University Press, 1995)

14. Turner, M. G., Gardner R. H., O’Neill, R. V. Landscape ecology in theory and practice. (Springer-Verlag New York, 2001).

15. Wiens, J. A., Schooley, R. L., \& Weeks, R. D. Patchy Landscapes and Animal Movements: Do Beetles Percolate? Oikos 78(2), 257-264 (1997).

16. Dolman, P. M., Hinsley, S. A., Bellamy, P. E., Watts. K. Woodland birds in patchy landscapes: the evidence base for strategic networks. Ibis 149, 146-160 (2007). 
17. Laiolo, P., Tella, J. L. Landscape bioacoustics allow detection of the effects of habitat patchiness on population structure, Ecology 87(5), 1203-1214 (2006).

18. Jia, Y., Tang, L., Xu, M., Yang, X. Landscape pattern indices for evaluating urban spatial morphology - A case study of Chinese cities. Ecological Indicators 99, 27-37 (2019).

19. Bosch, M. \& Chenal, J. Spatiotemporal patterns of urbanization in three Swiss urban agglomerations: insights from landscape metrics, growth modes and fractal analysis. Preprint at http://dx.doi.org/10.1101/645549 (2019).

20. Wang, H., Huang, Y., Wang, D., Chen, H. Effects of urban built-up patches on native plants in subtropical landscapes with ecological thresholds - A case study of Chongqing city. Ecological Indicators 108, 105751 (2020).

21. McGarigal, K., \& Cushman, S.A. The gradient concept of landscape structure in Issues and perspectives in landscape ecology (eds. Wiens, J., \& Moss, M.) 112-119 (Cambridge University Press, 2005).

22. Cushman, S. A., Gutzweiler, K., Evans, J., McGarigal, K. The Gradient Paradigm: A Conceptual and Analytical Framework for Landscape Ecology in Spatial Complexity, Informatics, and Wildlife Conservation (eds. Cushman, S. A. \& Huettmann, F.) 83-108. (Springer-Verlag Tokyo, 2010).

23. McGarigal, K., Tagil, S. \& Cushman, S.A. Surface metrics: an alternative to patch metrics for the quantification of landscape structure. Landscape Ecology 24(3) 433-450 (2009).

24. Fan, C., Myint, S. W., Rey, S.J., Li, W. Time series evaluation of landscape dynamics using annual Landsat imagery and spatial statistical modeling: Evidence from the Phoenix metropolitan region. International Journal of Applied Earth Observation and Geoinformation 58, 12-25. (2017).

25. Kowe, P., Mutanga, O., Odindi, J., Dube, T. A quantitative framework for analysing long term spatial clustering and vegetation fragmentation in an urban landscape using multi-temporal landsat data. International Journal of Applied Earth Observation and Geoinformation 88, 102057 (2020).

26. Wang, J., Kuffer, M. \& Pfeffer, K., The role of spatial heterogeneity in detecting urban slums. Computers, Environment and Urban Systems 73, 95-107 (2019).

27. Wu, D.Q. et al. Multi-Scale Identification of Urban Landscape Structure Based on Two-Dimensional Wavelet Analysis: The Case of Metropolitan Beijing, China. Ecological Complexity 43, 100832 (2020).

28. Rocchini, D. et al. Fourier transforms for detecting multitemporal landscape fragmentation by remote sensing. International Journal of Remote Sensing 34(24), 8907-8916 (2013).

29. Bennett, M.M. \& Smith, L.C. Advances in using multitemporal night-time lights satellite imagery to detect, estimate, and monitor socioeconomic dynamics. Remote Sensing of Environment 192, 176-197(2017).

30. Zhuo, L. et al. Modelling the population density of China at the pixel level based on DMSP/OLS non-radiancecalibrated night-time light images. International Journal of Remote Sensing 30(4), 1003-1018 (2009).

31. Zhang, Q. \& Seto, K.C. Mapping urbanization dynamics at regional and global scales using multi-temporal DMSP/OLS nighttime light data. Remote Sensing of Environment 115(9), 2320-2329 (2011.).

32. Yang, B. et al. A spatio-temporal method for crime prediction using historical crime data and transitional zones identified from nightlight imagery. International Journal of Geographical Information Science 34(9), 1740-1764 (2020). 
33. Chen, $X$. Nighttime Lights and Population Migration: Revisiting Classic Demographic Perspectives with an Analysis of Recent European Data. Remote Sensing 12(1), 169 (2020).

34. Li, X., Li, D., Xu, H., Wu, C. Intercalibration between DMSP/OLS and VIIRS night-time light images to evaluate city light dynamics of Syria's major human settlement during Syrian Civil War. International Journal of Remote Sensing 38(21), 5934-5951 (2017).

35. Duan X, Hu Q, Zhao P, Wang S, Ai M. An Approach of Identifying and Extracting Urban Commercial Areas Using the Nighttime Lights Satellite Imagery. Remote Sensing 12(6), 1029 (2020).

36. Krikigianni, E., Tsiakos, C. \& Chalkias, C. Estimating the relationship between touristic activities and night light emissions. European Journal of Remote Sensing 52(sup1),233-246 (2019).

37. Israeli Ministry of Health. Online geodatabase on COVID-19 exposures (In Hebrew) https://imoh.maps.arcgis.com/apps/webappviewer/index.html?id=20ded58639ff4d47a2e2e36af464c36e\&locale=he $\& 1$

38. Sharav, N. et al. Gush Dan Metro. The Economic, Social and Urban Impacts of the Gush Dan Metro Network Executive Report. Ministry of Finance, Ministry of Transport and Road Safety, National Economic Council Prime Minister's Office, NATA, Ayalon Highways, July 2020. (In Hebrew)

https://www.gov.il/BlobFolder/reports/metro_goshdan_jul_2020/he/Metro_Executive_Summary_14_july_2020.pdf

39. Moellering, H. \& Tobler, W. Geographical Variances. Geographical Analysis 4(1) 34-50 (1972).

40. Wu, J., Jelinski, D., Luck, M., Tueller, P. T. Multiscale Analysis of Landscape Heterogeneity: Scale Variance and Pattern Metrics. Annals of GIS 6(1) 6-19. (2000).

41 . Shen, W., Darrel Jenerette, G., Wu, J., Gardner, R. H. Evaluating empirical scaling relations of pattern metrics with simulated landscapes. Ecography 27(4) 459-469 (2004).

42. Wu, J. Effects of changing scale on landscape pattern analysis: scaling relations. Landscape Ecology 19(2), 125138 (2004).

43. Mahadevan, A. \& Campbell, J.W. Biogeochemical patchiness at the sea surface. Geophysical Research Letters 29(19), 32-1-32-4 (2002).

44. Yamamoto, D. Scales of regional income disparities in the USA, 1955 2003. Journal of Economic Geography 8(1), 79-103 (2007).

45. USGS Earth Explorer https://earthexplorer.usgs.gov

46. Román, M.O., et al. NASA's Black Marble nighttime lights product suite. Remote Sensing of Environment 210, 113-143 (2018)

47. Google Earth Engine Data Catalog, Earth Observation Group, Payne Institute for Public Policy, Colorado School of Mines.

https://developers.google.com/earth-engine/datasets/catalog/NOAA_VIIRS_DNB_MONTHLY_V1_VCMCFG 


\section{Figures}

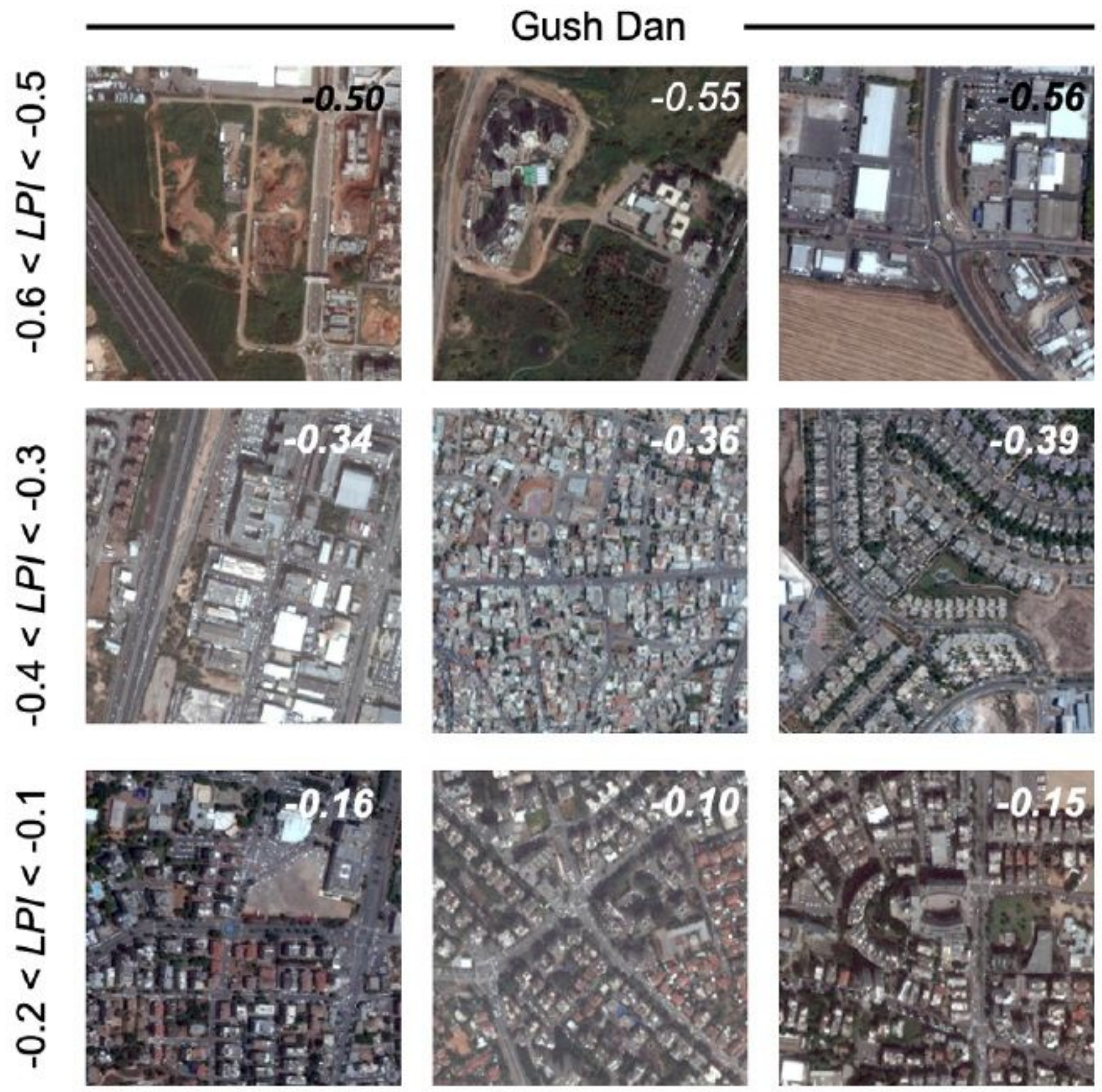

Figure 1

Examples of the variability of landscape types across different ranges captured by LPI for the area of Gush Dan District. It is shown a true color image of LPI ranges within the interval $-0.1<\mathrm{LPI}<-0.6$ which demonstrates the captured differences in urban landscape heterogeneity. 
SARS-CoV-2 Exposures based on Human Activity type

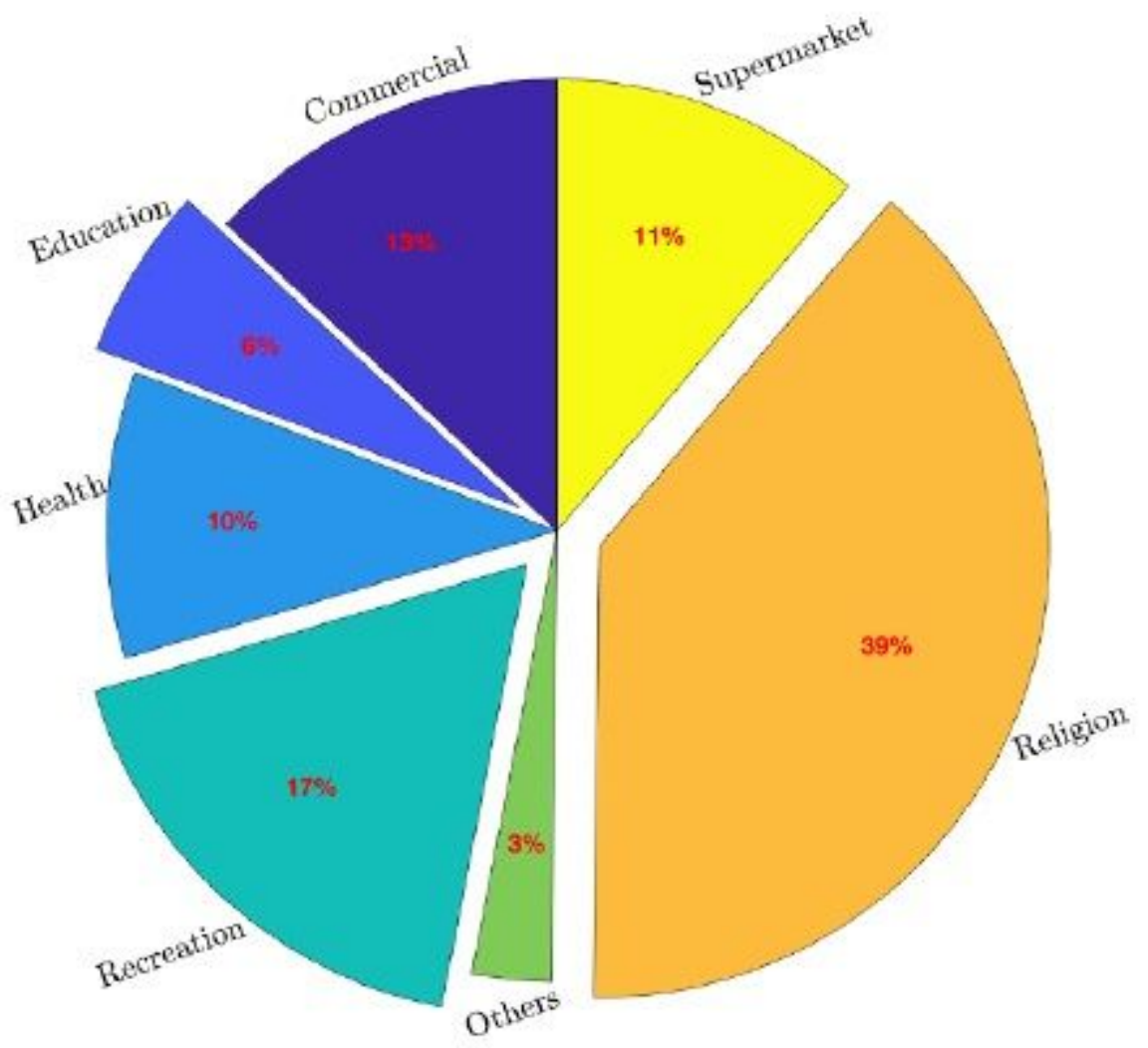

\section{Figure 2}

Pie chart describing the classification of RLSCs into human activity types. 
True color image and Exposures

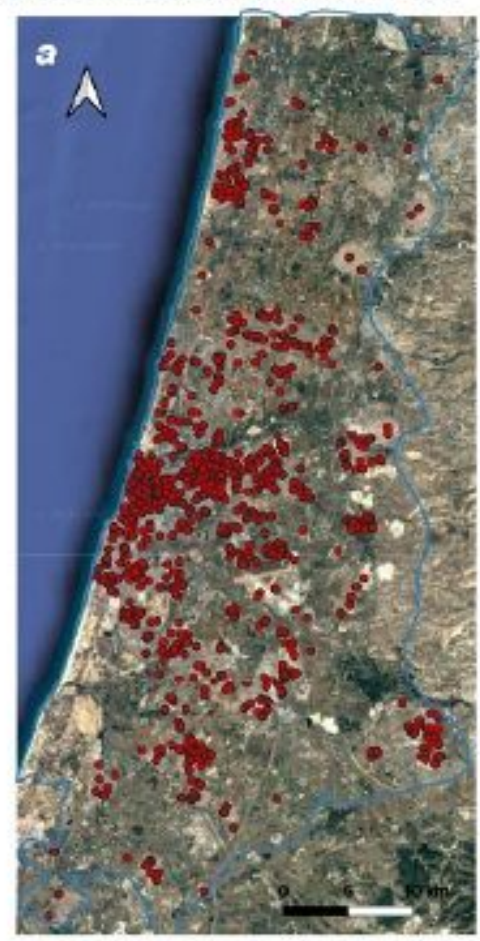

NTL and Exposures

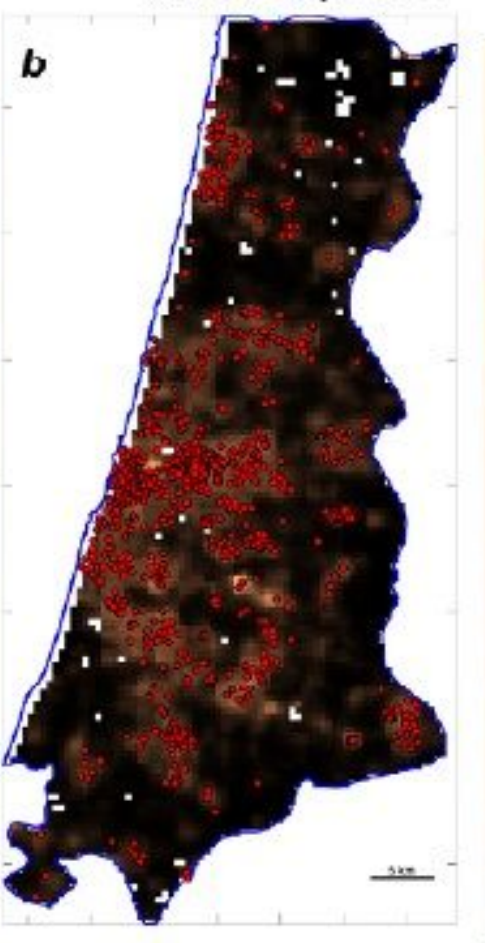

LPI and Exposures

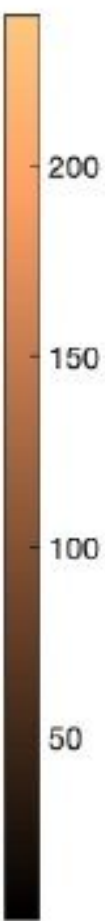

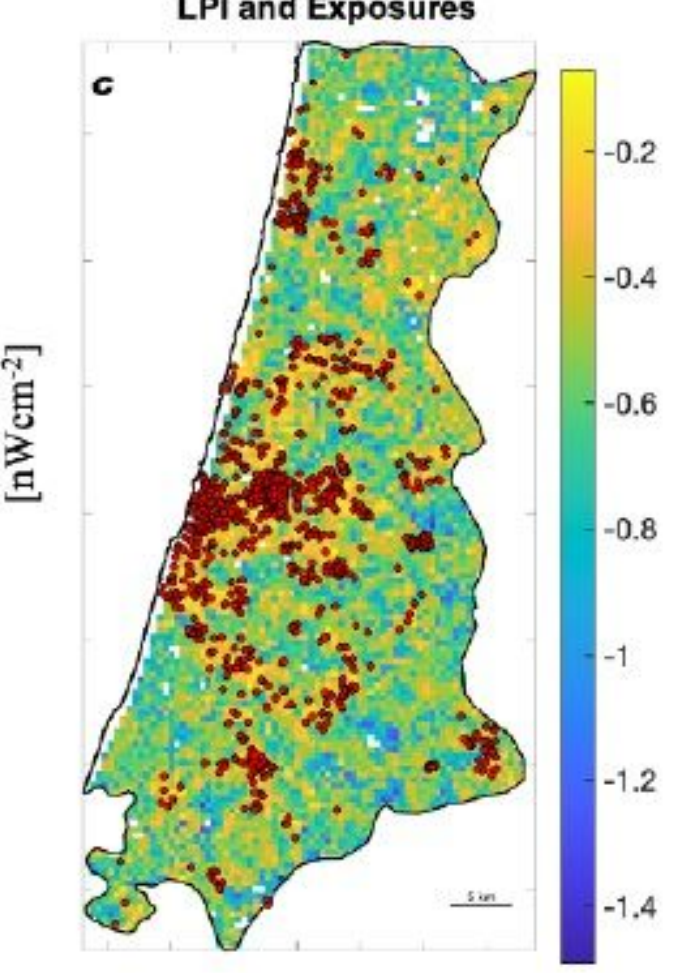

Figure 3

Spatial distribution of RLSC in Gush Dan District. RLSC in Gush Dan (red points), overlaid on (a) a truecolor satellite image (Gush Dan borders in blue); (b) NTL map; (c) LPI map. Note that RLSCs preferentially occur in regions with high LPI values, which correspond to regions with high NTL values. 


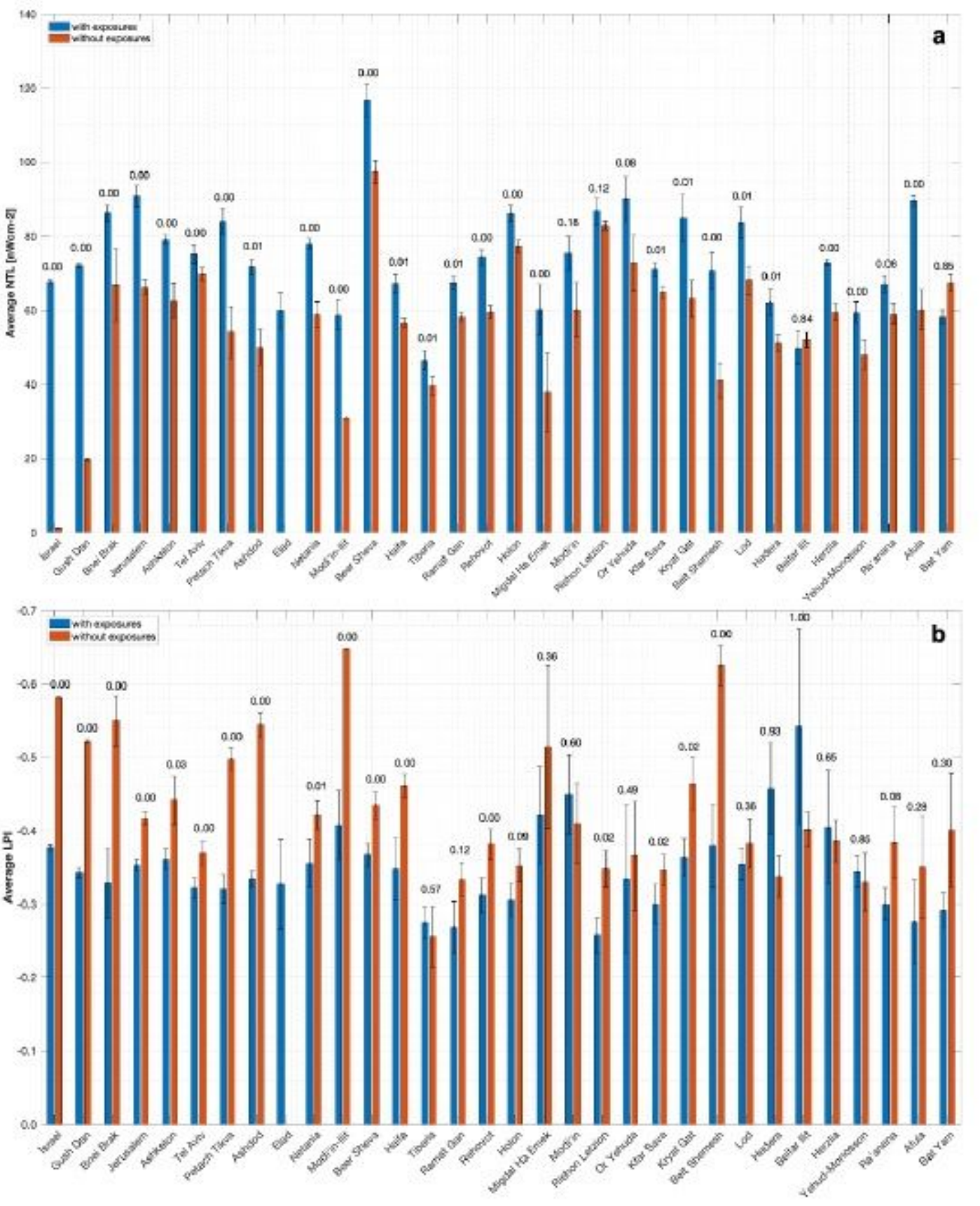

Figure 4

NTL and LPI statistics for our sample. Bar plot showing the mean of (a) NTL and (b) LPI in grid cells with exposures (blue bars) and without exposures (red bars) for the 30 cities with more than 100 reported RLSC cases. For comparison we also present the statistics for the full sample of cities, as well as that for the Gush Dan conurbation. Note that in almost all of the cities both NTL and LPI are significantly higher at locations with finite RLSC values. 


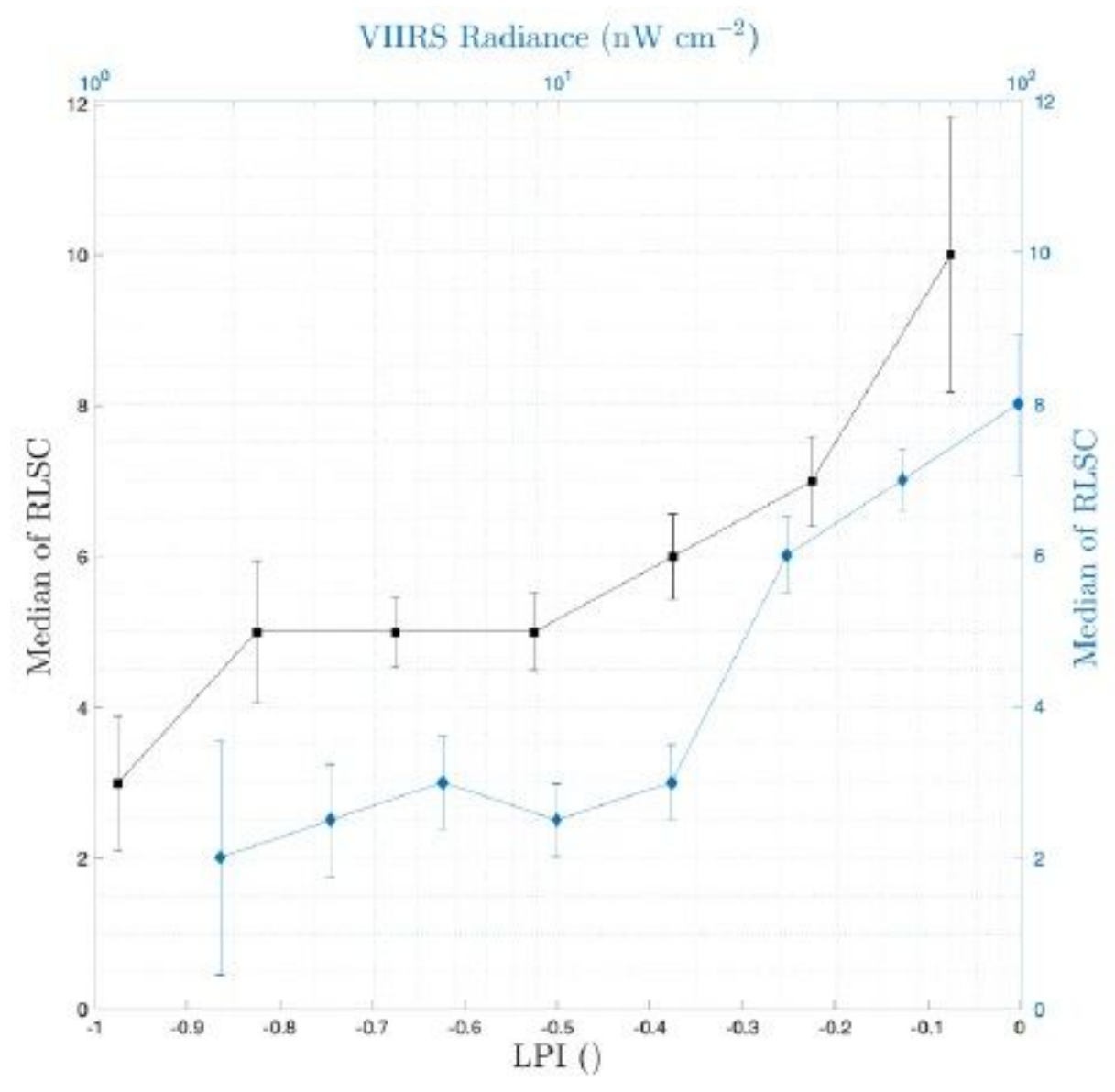

Figure 5

Correlation between RLSC and NTL (blue curve) and LPI (line curve). Dots and error-bars denote the median and standard deviation in each bin. Note the qualitatively similar behavior for the NTL and LPI statistics demonstrating the commonalities between LPI and NTL as tracers of human activity andlandscape development. 